\title{
EDITORIAL
}

\section{Investigación para sustentar políticas farmacéuticas}

$\mathrm{D}$ e acuerdo con la Organización Mundial de la Salud (OMS), los objetivos principales de una política farmacéutica nacional son la disponibilidad y el acceso equitativo a medicamentos con precios asequibles, su calidad, seguridad y eficacia y el uso adecuado y eficiente de los medicamentos por parte de los profesionales del cuidado de la salud y los consumidores. ${ }^{1}$ Alcanzar todos estos objetivos al mismo tiempo no es fácil para ningún país -como lo ha mostrado la experiencia alrededor del mundo-, pero al surgir la investigación sobre política farmacéutica como disciplina se espera que sus estudios generen conocimiento respecto de la creación de políticas para alcanzar estos objetivos en diferentes contextos. Esta edición especial de Salud Pública de México presenta trabajos de investigación realizados recientemente por México y América Latina en esta área relacionados particularmente con la situación mexicana, para promover el cumplimiento de los objetivos de política farmacéutica y de salud pública.

No hay duda de que el tema de la política farmacéutica se ubica actualmente entre las más altas prioridades del sector salud mexicano. México está en un proceso de reforma importante: la creación de un nuevo esquema de seguro (Seguro Popular) para la mitad de la población que no había sido previamente asegurada. La meta es proveer de seguro médico a todos los mexicanos para 2010. Esta reforma política de salud ha tenido un gran impacto sobre la política farmacéutica: está incrementando significativamente el gasto en medicamentos, demanda la creación de una nueva infraestructura para la adquisición y suministro de medicamentos al nivel federal así como en los estados. Asimismo, requiere de un incremento en recursos humanos y de capacitación de los mismos para prescribir y proveer los medicamentos de forma racional, de manera que se logre un impacto positivo en la salud de la población recientemente asegurada. Uno de los mayores retos de la reforma es la creación de mecanismos que permitan fomentar la transparencia y la rendición de cuentas, que son dos elementos de una buena rectoría que asegura la sustentabilidad del programa.

El gobierno mexicano inició la discusión sobre una reforma de la política farmacéutica en el pasado sexenio. En 2005, publicó el documento oficial titulado "Hacia una política farmacéutica integral para México", el cual comienza a describir los diferentes aspectos necesarios para una nueva política farmacéutica en el país. Este fue un paso histórico en la política pública. Fue el primer documento multifacético sobre política farmacéutica en México por décadas. El documento proporciona las bases para una discusión seria entre grupos de interés en el sector farmacéutico.

Sin embargo, hacer una evaluación acertada de la política farmacéutica mexicana actual resulta difícil por varias razones. En primer lugar, han ocurrido cambios simultáneos en el diseño e implementación de diversas estrategias descritas en el documento desde su publicación. En este contexto, es interesante hacer notar que a pesar del hecho de que el documento ha sido definido como una postura oficial, una propuesta, y no como la política farmacéutica actual, un número sustancial de tomadores de decisiones lo ha percibido erróneamente como la nueva política farmacéutica. Ésta todavía no existe. En segundo lugar, los cambios políticos han evolucionado rápidamente, con pocas oportunidades para consultar a los grupos de interés y con poca transparencia. En tercer lugar, la información necesaria para evaluar el desempeño de la política farmacéutica en México no está en el dominio público y no ha sido analizada sistemáticamente. Finalmente, no se ha realizado en México un debate abierto sobre los diferentes objetivos de la política farmacéutica, así como tampoco se ha llevado a cabo una discusión abierta sobre la importancia relativa de los diferentes objetivos.

México no es el único país que está en el proceso de repensar su política nacional farmacéutica ni el único con problemas para crear un debate abierto sobre dicha política. Es importante anotar que existen muchas 
iniciativas globales en camino que impulsan el diseño de las políticas farmacéuticas y que pueden apoyar a México en su esfuerzo para reformar su política. El reto continuo del acceso universal y sostenible de terapia antirretroviral para el tratamiento del VIH/SIDA ha traído a la agenda política global de salud un problema más grande: el acceso a medicamentos en general. Se ha dicho que el acceso a medicamentos es un derecho humano básico. En 2005, los líderes globales se comprometieron a apoyar los esfuerzos para mejorar el acceso al tratamiento. ${ }^{2}$ Una de las metas del milenio supone proveer de acceso a medicamentos en colaboración con la industria farmacéutica. ${ }^{3}$

Varias nuevas iniciativas internacionales buscan ahora formas innovadoras para alcanzar la meta de mejorar el acceso a los medicamentos. Estas iniciativas brindan especial atención a los derechos de propiedad intelectual, el precio de los medicamentos esenciales y la transparencia y rendición de cuentas. Dos de estas iniciativas merecen especial atención aquí:

1. La Asamblea Mundial de Salud aprobó en mayo de 2008 una resolución para una estrategia global y un plan de acción en salud pública, innovación y derechos de propiedad intelectual. ${ }^{4}$ El plan fue desarrollado por el Grupo de Trabajo Intergubernamental de la OMS entre 2006 y 2008, analizando métodos para fomentar la innovación y el mejoramiento del acceso a medicamentos para poder obtener mejores resultados en salud, en los países en desarrollo.

2. El Departamento de Desarrollo Internacional del Reino Unido (DFID) ha iniciado una nueva iniciativa, Medicines Transparency Alliance (MeTA), para apoyar los esfuerzos nacionales de aumentar la transparencia en el sector farmacéutico y construir una política de capacitación en la adquisición y el abastecimiento de medicamentos. ${ }^{5}$ Además, MeTA fortalecerá el desarrollo e implementación de estrategias políticas nacionales para incrementar la transparencia y rendición de cuentas en el sector farmacéutico. MeTA utiliza un proceso de multigrupos de interés y ha recibido apoyo de varias organizaciones, incluyendo la Organización Mundial de la Salud, el Banco Mundial, la Federación Internacional de la Industria Farmacéutica (IFPMA) y la Acción Internacional para la Salud/Health Action International (HAI).

Estas dos iniciativas muestran la relevancia global de las políticas nacionales de acceso aunadas con la transparencia y la rendición de cuentas, y tienen una importancia particular en el caso de México.
Actualmente en México hay dos cambios importantes de la política farmacéutica en proceso: primero, la anulación del requisito de tener una planta para poder importar medicamentos genéricos; este cambio tiene la posibilidad de mejorar la competencia en el mercado farmacéutico; y segundo, el establecimiento de una comisión interinstitucional que tratará de coordinar la adquisición de medicamentos patentados en los diferentes sistemas, que podría resultar en mejorar los procesos de compra entre IMSS, ISSSTE y Seguro Popular y en crear más valor por el gasto ejercido. Ciertamente, la política farmacéutica mexicana necesita más cambios para poder respaldar los objetivos más amplios de la salud pública. Sin embargo, la política farmacéutica es reconocida ahora por los niveles más altos del gobierno mexicano, incluyendo al ejecutivo, como un área principal de la reforma que facilitará cambios futuros.

Este número especial de Salud Pública de México se propone presentar parte de la evidencia de investigación que ha sido recolectada en los últimos años, la que provee lecciones importantes para el diseño e implementación de políticas en el contexto mexicano y latinoamericano. Se espera que su publicación estimule la investigación en el área de política farmacéutica y también la discusión pública en México sobre los cambios requeridos en aquellas políticas públicas que afectan los medicamentos y la salud.

\section{Dra. Veronika J Wirtz, ${ }^{(1)}$ \\ Dr. Michael R Reich, ${ }^{(2)}$ \\ Dr. Mauricio Hernández-Ávila, ${ }^{(3)}$ \\ (1) Profesora-Investigadora,}

Centro de Investigación de Sistemas de Salud, Instituto Nacional de Salud Pública. México.

(2) Professor, Harvard School of Public Health, Harvard University. USA.

(3) Subsecretario de Prevención y Promoción de la Salud, Secretaría de Salud. México.

\section{Referencias}

I. World Health Organization. How to develop and implement a national drug policy. 2nd edition. Geneva:World Health Organization, 2003. 2. Department of International Development (DFID). G8 Gleneagles: One year on-turning talk into action. London: HM Goverment, 2006. 3. United Nations Millennium Development Goals. Goals Target 4, New York; US: United Nations, $200 \mathrm{I}$.

4. World Health Assembly. Global strategy and plan of action on public health, innovation and intellectual property.WHA6I.2I.Agenda item II.6. 5. Short R. UK leads initiative to drive down cost of drugs in poor countries. BMJ 2007;334:870. 